\title{
Sub-Additive Aggregation Functions and Their Applications in Construction of Coherent Upper Previsions
}

\author{
Serena Doria ${ }^{1}\left(\mathbb{0}\right.$, Radko Mesiar $^{2}\left(\mathbb{C}\right.$ and Adam Šeliga ${ }^{2, *}$ (D) \\ 1 Department of Engineering and Geology, University G. d'Annunzio, 66100 Chieti-Pescara, Italy; \\ serena.doria@unich.it \\ 2 Faculty of Civil Engineering, Slovak University of Technology, Radlinského 11, 81005 Bratislava, Slovakia; \\ radko.mesiar@stuba.sk \\ * Correspondence: adam.seliga@stuba.sk
}

Citation: Doria, S.; Mesiar, R.; Šeliga, A. Sub-Additive Aggregation Functions and Their Applications in Construction of Coherent Upper Previsions. Mathematics 2021, 9, 2. https://dx.doi.org/10.3390/ math 9010002

Received: 8 November 2020 Accepted: 20 December 2020 Published: 22 December 2020

Publisher's Note: MDPI stays neutral with regard to jurisdictional claims in published maps and institutional affiliations.

Copyright: () 2020 by the authors. Licensee MDPI, Basel, Switzerland. This article is an open access article distributed under the terms and conditions of the Creative Commons Attribution (CC BY) license (https: / / creativecommons.org/ licenses/by/4.0/).

\begin{abstract}
In this paper, we explore the use of aggregation functions in the construction of coherent upper previsions. Sub-additivity is one of the defining properties of a coherent upper prevision defined on a linear space of random variables and thus we introduce a new sub-additive transformation of aggregation functions, called a revenue transformation, whose output is a sub-additive aggregation function bounded below by the transformed aggregation function, if it exists. Method of constructing coherent upper previsions by means of shift-invariant, positively homogeneous and sub-additive aggregation functions is given and a full characterization of shift-invariant, positively homogeneous and idempotent aggregation functions on $\left[0, \infty\left[{ }^{n}\right.\right.$ is presented. Lastly, some concluding remarks are added.
\end{abstract}

Keywords: sub-additivity; revenue transformation; aggregation function; coherent upper prevision

\section{Introduction}

In the subjective probability approach [1], prevision of a random variable is defined by the notion of coherence that assures that the prevision of a random variable $X$ is the amount the subject is willing to bet on $X$ such that neither the bettor nor the banker can win or loose for sure. From a mathematical point of view, linear previsions on a linear space of random variables are coherent if and only if they are linear functionals with values bounded by the infimum and the supremum value of the random variable. From these defining properties, we can prove that a linear coherent prevision is a homogeneous functional. Coherent probabilities are obtained when only indicator functions are considered in the domain. Because of the incomplete and inaccurate information, or to represent some preference orderings (e.g., Example 1 of [2]), in some cases it is appropriate to consider non-linear functionals. Coherent upper and lower previsions can be defined as generalizations of linear previsions. In particular, if a linear prevision is defined with respect to a countably additive probability, coherent upper and lower conditional previsions can be defined by the Choquet integral with respect to the outer and inner measures generated by the countably additive probability, which are the natural extensions of an additive probability defined on a $\sigma$-field [3]. A new model of coherent upper previsions defined by the Choquet integral with respect to Hausdorff outer measures is proposed in [4]. Also, coherent upper and lower previsions have a behavioural interpretation: the lower prevision of $X$ can be regarded as the supremal buying price for the random variable $X$ and the upper prevision is an infimal selling price. Nevertheless, we observe that there exist coherent upper previsions defined on the linear space of random variables without linear restrictions; an example is the vacuous upper prevision of a random variable, defined as the supremum of the values assumed by that random variable on $\Omega$. So an open problem is to propose new mathematical tools to define coherent upper previsions that cannot be obtained as extensions of linear previsions. 
The main motivation behind this paper is to propose a construction method for coherent upper previsions using aggregation functions $[5,6]$ that are prolific and latter-day part of mathematics that found its place both in the theory and applications.

A functional on a linear space of random variables is a coherent upper prevision if and only if it is bounded by the supremum value of the random variable, sub-additive and positively homogeneous. In this contribution, coherent upper previsions are constructed by aggregation functions, which are interpreted as the gains we obtain with a given resource so that the increase in gains, after increase in resources, can be expressed in terms of aggregation functions. We introduce a new type of transformation of aggregation functions, called a revenue transformation, which represents the best upper bound for possible gains; in the paper we consider only revenue transformations that always assume bounded values. Some properties of revenue transformations are proven; in particular, it is proven that the revenue transformation transforms any aggregation function to some other aggregation function that is sub-additive and is bounded belowed by the transformed function. There already exists a sub-additive transformation of aggregation functions introduced in [7] and is heavily studied by researchers [8-10]. This sub-additive transformation always exists and is bounded above by the aggregation function that is being transformed, whereas examples are given in this paper of aggregation functions that do not have a bounded revenue transformation.

The revenue transformation of the Choquet integral, which is an example of aggregation function, is calculated and an open problem is to determine if it is the Choquet integral with respect to a monotone measure. An example of revenue transformation of Choquet integral with respect to a monotone measure $\mu$, which is not sub-modular, is given such that the transformation is the Choquet integral with respect to a sub-modular monotone measure and it can be used to define a coherent upper prevision.

The paper is organized as follows. In Section 2, some basic preliminaries needed later are given. In Section 3, we define a new sub-additive transformation of aggregation functions, called a revenue transformation, and study its properties. Section 4 consists of a construction method of coherent upper previsions based on aggregation functions. This construction method is heavily exemplified in this section. The last section contains some concluding remarks on the topic.

\section{Preliminaries}

Let $\Omega$ be a non-empty set and let $\mathcal{A}$ be a $\sigma$-algebra on $\Omega$ such that $\mathcal{A}$ is finite. With this assumption we may, without loss of generality, assume that $\Omega=\{1,2, \ldots, n\}$ and $\mathcal{A}=2^{\Omega}$ for some $n \in \mathbb{N}$. This natural number $n$ is fixed throughout. Note that the motivation of the concepts discussed in this paper given in the literature, see, e.g., [3], including illustrative examples, deal mostly with finite $\Omega$ only, therefore we focus on this framework only. Though most of concepts we recall or introduce in this paper remain valid also when a general measurable space $(\Omega, \mathcal{A})$ is considered, this is not the case, e.g., with the constructions based on the aggregation functions.

In this setting, every random variable $X: \Omega \rightarrow \mathbb{R}$ on $(\Omega, \mathcal{A})$ can be represented as $n$-tuple $\mathbf{x} \in \mathbb{R}^{n}$ with $i$-th coordinate (denoted by $x_{i}$ ) being equal to $X(i)$ for $i=1,2, \ldots, n$. All such vectors (i.e., random variables) form a vector space $\mathbb{R}^{n}$ with coordinate-wise addition + and coordinate-wise scalar multiplication (over field $\mathbb{R}$ ). Note that coordinatewise difference -, coordinate-wise multiplication *, coordinate-wise division /, coordinatewise supremum $\vee$, and coordinate-wise infimum $\wedge$ can be also introduced.

Also, a partial order $\leq$ can be introduced by $\mathbf{x} \leq \mathbf{y}$ if and only if $x_{i} \leq y_{i}$ for all $i \in\{1,2, \ldots, n\}$ and all $\mathbf{x}, \mathbf{y} \in \mathbb{R}^{n}$. By $\mathbf{0}$ we denote the all-zero vector and by $\mathbf{1}$ we denote the all-one vector. An indicator vector $\mathbf{1}_{A}$ of a set $A \subseteq \Omega$ is given by its $i$-th coordinate being equal to $\mathbf{1}_{A}(i)$, where $\mathbf{1}_{A}$ is the indicator function of the set $A$. A positive cone of a vector space $\mathbb{R}^{n}$ is the set

$$
\mathbb{R}_{+}^{n}=\left\{\mathbf{x} \in \mathbb{R}^{n}: \mathbf{x} \geq \mathbf{0}\right\},
$$

i.e., $\mathbb{R}_{+}^{n}=\left[0, \infty{ }^{n}\right.$. 
Let $\mathbf{x} \in \mathbb{R}^{n}$ be any vector with coordinates $x_{i}$. There exists a permutation $\sigma: \Omega \rightarrow \Omega$ such that

$$
x_{\sigma(1)} \leq x_{\sigma(2)} \leq \cdots \leq x_{\sigma(n)} .
$$

For simplicity, we denote $x_{(1)}=x_{\sigma(1)}, x_{(2)}=x_{\sigma(2)}, \ldots, x_{(n)}=x_{\sigma(n)}$. Thus we have $\wedge_{i} x_{i}=x_{(1)}$ and $\vee_{i} x_{i}=x_{(n)}$. Note that $x_{(i)}$ is just the $i$-th order statistics of the sample $\left(x_{1}, \ldots, x_{n}\right)$.

An aggregation function $[5,6]$ is any mapping $A: \mathbb{R}_{+}^{n} \rightarrow[0, \infty[$ such that $A$ is nondecreasing, i.e., $\mathbf{0} \leq \mathbf{x} \leq \mathbf{y}$ implies $A(\mathbf{x}) \leq A(\mathbf{y})$; and grounded, i.e., $A(\mathbf{0})=0$. In practice, it is convenient to assume that there exists at least one $\mathbf{x} \in \mathbb{R}_{+}^{n}$ such that $A(\mathbf{x}) \neq 0$. This restriction is not necessary, but $A(\mathbf{x}) \equiv 0$ is not an interesting aggregation function for us and thus such case will be omitted in proofs (with explicit declaration). We say that an aggregation function $A$ is sub-additive, respectively super-additive, if and only if

$$
A(\mathbf{x}+\mathbf{y}) \leq A(\mathbf{x})+A(\mathbf{y}), \quad \text { respectively } A(\mathbf{x}+\mathbf{y}) \geq A(\mathbf{x})+A(\mathbf{y}),
$$

holds for all $\mathbf{x}, \mathbf{y} \in \mathbb{R}_{+}^{n}$. We say that an aggregation function $A$ is positively homogeneous if and only if

$$
A(\alpha \mathbf{x})=\alpha A(\mathbf{x})
$$

for all $\mathbf{x} \in \mathbb{R}_{+}^{n}$ and all $\alpha \geq 0$. Lastly, we say that an aggregation function $A$ is shift-invariant if and only if

$$
A(\mathbf{x}+\alpha \mathbf{1})=A(\mathbf{x})+\alpha A(\mathbf{1})
$$

for all $\mathbf{x} \in \mathbb{R}_{+}^{n}$ and all $\alpha \geq-\inf \mathbf{x} . \mathrm{A}[0,1]$-aggregation function is any mapping $A:[0,1]^{n} \rightarrow$ $[0,1]$ such that $A$ is non-decreasing, i.e., $\mathbf{0} \leq \mathbf{x} \leq \mathbf{y} \leq \mathbf{1}$ implies $A(\mathbf{x}) \leq A(\mathbf{y})$; and obeys conditions $A(\mathbf{0})=0$ and $A(\mathbf{1})=1$. Sub-additivity, super-additivity, positive homogeneity, and shift-invariance can be introduced analogously for $[0,1]$-aggregation functions.

Note that a full characterization of positively homogenenous and shift-invariant $[0,1]$ aggregation functions was given in [6] (Proposition 7.37): A $[0,1]$-aggregation function $A$ is shift-invariant and positively homogeneous (interval scale invariant in [6]) if and only if $A$ satisfies the following conditions:

(i) $\quad A(\mathbf{x})-A(\mathbf{y}) \leq \vee_{i}\left(x_{i}-y_{i}\right)$ for all $\mathbf{x}, \mathbf{y} \in[0,1]^{n}$ such that $\mathbf{x} \geq \mathbf{y}$ and $x_{i}=y_{i}=0$ for some $i \in\{1,2, \ldots, n\}$;

(ii) $\quad A(\mathbf{x}) / A(\mathbf{y}) \geq \wedge_{i}\left(x_{i} / y_{i}\right)$ for all $\mathbf{x}, \mathbf{y} \in[0,1]^{n}$ such that $\mathbf{x} \leq \mathbf{y}$ and $x_{j}=y_{j}=1$ for some $j \in\{1,2, \ldots, n\}$, with convention $0 / 0=1$.

Observe that then

$$
A(\mathbf{x})=\left(\vee_{i} x_{i}-\wedge_{i} x_{i}\right) A\left(\frac{\mathbf{x}-\left(\wedge_{i} x_{i}\right) \mathbf{1}}{\vee_{i} x_{i}-\wedge_{i} x_{i}}\right)+\wedge_{i} x_{i}=\left(x_{(n)}-x_{(1)}\right) A\left(\frac{\mathbf{x}-x_{(1)} \mathbf{1}}{x_{(n)}-x_{(1)}}\right)+x_{(1)}
$$

for all $\mathbf{x}$ such that $\vee_{i} x_{i} \neq \wedge_{i} x_{i}$ and

$$
A(x \mathbf{1})=x
$$

for all $x \in[0,1]$. See also, e.g., [11,12].

A monotone measure [13] is any set function $\mu: \mathcal{A} \rightarrow[0, \infty[$ that is non-decreasing, i.e., $A \subseteq B \subseteq \Omega$ implies $\mu(A) \leq \mu(B)$; and grounded, i.e., $\mu(\varnothing)=0$. Note that a monotone measure can be viewed as an aggregation function defined on $2^{\Omega}$ with the partial order $\leq$ being set inclusion. We say that a monotone measure $\mu$ is sub-modular if and only if

$$
\mu(A \cup B)+\mu(A \cap B) \leq \mu(A)+\mu(B)
$$

for all $A, B \in 2^{\Omega}$. Sub-modular monotone measures are also sometimes referred to as 2-alternating monotone measures, see, e.g., [14,15]. If $A=\{x, y, \ldots, z\} \in 2^{\Omega}$ we will write, 
for simplicity, $\mu_{x y \ldots z}$ instead of $\mu(\{x, y, \ldots, z\})$ or $\mu_{A}$ instead of $\mu(A)$. A conjugate monotone measure for a monotone measure $\mu$ is a monotone measure $\bar{\mu}: 2^{\Omega} \rightarrow[0, \infty[$ such that

$$
\bar{\mu}(A)=\mu(\Omega)-\mu(\Omega \backslash A)
$$

for all $A \in 2^{\Omega}$.

The Choquet integral [16] with respect to a monotone measure $\mu$ is the operator $\mathrm{Ch}^{\mu}: \mathbb{R}_{+}^{n} \rightarrow[0, \infty[$ such that

$$
\mathrm{Ch}^{\mu}(\mathbf{x})=x_{(1)} \mu_{A_{1}}+\sum_{i=2}^{k}\left(x_{(i)}-x_{(i-1)}\right) \mu_{A_{i}},
$$

where $A_{i}=\left\{j \in \Omega: x_{j} \geq x_{(i)}\right\}$ for $i=1,2, \ldots, n$. Choquet integral is an aggregation function and if $\mu$ is sub-modular then $\mathrm{Ch}^{\mu}$ is a sub-additive aggregation function. In the theory of imprecise probabilities, an extension of Choquet integral, called an asymmetric Choquet integral, that is an operator $\mathrm{aCh}^{\mu}: \mathbb{R}^{n} \rightarrow \mathbb{R}$ given by

$$
\mathrm{aCh}^{\mu}(\mathbf{x})=\mathrm{Ch}^{\mu}(\mathbf{x} \vee \mathbf{0})-\mathrm{Ch}^{\bar{\mu}}((-\mathbf{x}) \vee \mathbf{0}),
$$

is used to construct coherent upper previsions, see, e.g., [17].

A coherent upper prevision is a mapping $\bar{P}: \mathbb{R}^{n} \rightarrow \mathbb{R}$ such that conditions

(CUP1) $\quad \bar{P}(\mathbf{x}) \leq \sup \mathbf{x}$ for all $\mathbf{x} \in \mathbb{R}^{n} ;$

(CUP2) $\quad \bar{P}(\alpha \mathbf{x})=\alpha \bar{P}(\mathbf{x})$ for all $\mathbf{x} \in \mathbb{R}^{n}$ and all $\alpha \in[0, \infty[;$ and

(CUP3) $\quad \bar{P}(\mathbf{x}+\mathbf{y}) \leq \bar{P}(\mathbf{x})+\bar{P}(\mathbf{y})$ for all $\mathbf{x}, \mathbf{y} \in \mathbb{R}^{n}$

hold. Note that condition (CUP2) is positive homogeneity of $\bar{P}$ and condition (CUP3) represents sub-additivity of $\bar{P}$. A coherent lower prevision is any mapping $\underline{P}: \mathbb{R}^{n} \rightarrow \mathbb{R}$ such that $\underline{P}(\mathbf{x})=-\bar{P}(-\mathbf{x})$ for all $\mathbf{x} \in \mathbb{R}^{n}$, where $\bar{P}$ is some coherent upper prevision.

\section{Revenue Transformation and Its Properties}

Let us start with a notion of a revenue transformation. Imagine that the value of an aggregation function $A: \mathbb{R}_{+}^{n} \rightarrow\left[0, \infty\left[\right.\right.$ at $\mathbf{y} \in \mathbb{R}_{+}^{n}$ represents gains with resources represented by $\mathbf{y}$. The increase in gains after increasing resources by $\mathbf{x}$ is equal to

$$
A(\mathbf{x}+\mathbf{y})-A(\mathbf{y})
$$

i.e., the difference between gains with increased resources and original resources. Imagine, that the original resources are not known and we ask ourselves a question: What is the maximal gain (or the best upper bound for possible gains) if we increase our resources by $\mathbf{x}$ ? The answer is

$$
\sup _{\mathbf{y} \geq \mathbf{0}}(A(\mathbf{x}+\mathbf{y})-A(\mathbf{y})) .
$$

This defines the revenue transformation of $A$ that will be denoted by $\bar{A}$. We would like to ensure that $\bar{A}$ is again an aggregation function and thus we must prevent $\bar{A}$ to being equal to $\infty$. Such value is obtainable, see following example.

Example 1. Consider a one-dimensional aggregation function $A:[0, \infty[\rightarrow[0, \infty[$ given by

$$
A(x)=x^{2}
$$

Then one obtains that

$$
A(x+y)-A(y)=(x+y)^{2}-y^{2}=x^{2}+2 x y
$$


and thus

$$
\sup _{y \geq 0}\left(2 x y+y^{2}\right)=\infty,
$$

for any $x>0$. As an example for $n$-dimensional aggregation function that has a similar behavour is an aggregation function $A: \mathbb{R}_{+}^{n} \rightarrow\left[0, \infty\left[: \mathbf{x} \mapsto x_{1}^{2}+x_{2}^{2}+\cdots+x_{n}^{2}\right.\right.$.

One of the existence conditions (and we will prove later that it is the only condition) for $\bar{A}$ to be an aggregation function is

$$
\sup _{\mathbf{y} \geq \mathbf{0}}(A(\mathbf{x}+\mathbf{y})-A(\mathbf{y}))<\infty
$$

that is required to hold for all $\mathbf{x} \in \mathbb{R}_{+}^{n}$. This defines a sub-class of aggregation functions that will be denoted by $\mathbb{A}_{\text {rev. }}$. Whenever we refer to any revenue transformation we will always assume that this condition is satisfied and thus we will only work with $A \in \mathbb{A}_{\text {rev }}$ in such a case.

Definition 1. Let $A: \mathbb{R}_{+}^{n} \rightarrow\left[0, \infty\left[\right.\right.$ be an aggregation function such that $A \in \mathbb{A}_{\text {rev. }}$ Then its revenue transformation is a mapping $\bar{A}: \mathbb{R}_{+}^{n} \rightarrow[0, \infty[$ given by

$$
\bar{A}(\mathbf{x})=\sup _{\mathbf{y} \geq \mathbf{0}}(A(\mathbf{x}+\mathbf{y})-A(\mathbf{y}))
$$

for all $\mathbf{x} \in \mathbb{R}_{+}^{n}$.

As we hinted before, any revenue transformation of any aggregation function belonging to $\mathbb{A}_{\text {rev }}$ yield again an aggregation function. This is stated in the following proposition.

Proposition 1. Let $A: \mathbb{R}_{+}^{n} \rightarrow\left[0, \infty\left[\right.\right.$ be an aggregation function from $\mathbb{A}_{\text {rev. }}$. Then $\bar{A}$ is also an aggregation function.

Proof. Let us start by proving that $\bar{A}(\mathbf{0})=0$ holds. It is easy to see that

$$
\bar{A}(\mathbf{0})=\sup _{\mathbf{y} \in \mathbb{R}_{+}^{n}}(A(\mathbf{0}+\mathbf{y})-A(\mathbf{y}))=\sup _{\mathbf{y} \in \mathbb{R}_{+}^{n}}(A(\mathbf{y})-A(\mathbf{y}))=\sup _{\mathbf{y} \in \mathbb{R}_{+}^{n}} 0=0 .
$$

Now we will show that $\bar{A}$ is also non-decreasing. Let $\mathbf{x}, \mathbf{z} \in \mathbb{R}_{+}^{n}$ be such that $\mathbf{x} \leq \mathbf{z}$. From the monotonicity of $A$ we have $A(\mathbf{x}+\mathbf{y}) \leq A(\mathbf{z}+\mathbf{y})$, or, equivalently, $A(\mathbf{x}+\mathbf{y})-$ $A(\mathbf{y}) \leq A(\mathbf{z}+\mathbf{y})-A(\mathbf{y})$ for any $\mathbf{y} \in \mathbb{R}_{+}^{n}$ and thus

$$
\bar{A}(\mathbf{x})=\sup _{\mathbf{y} \in \mathbb{R}_{+}^{n}}(A(\mathbf{x}+\mathbf{y})-A(\mathbf{y})) \leq \sup _{\mathbf{y} \in \mathbb{R}_{+}^{n}}(A(\mathbf{z}+\mathbf{y})-A(\mathbf{y}))=\bar{A}(\mathbf{z}) .
$$

Thus, $\bar{A}$ is an aggregation function.

Now we give some properties of revenue transformations. These are summarized in the following proposition.

Proposition 2. Let $A$ be an aggregation function. Then

a $\bar{A}$ is a sub-additive aggregation function;

b $\bar{A} \geq A$;

c if $A$ is sub-additive then $\bar{A}=A$;

$d$ if $A$ is shift-invariant then the diagonal of $\bar{A}$ coincides with the diagonal of $A$;

$e$ if $A$ is shift-invariant then so is $\bar{A}$; 
$f$ if $A$ is positively homogeneous then so is $\bar{A}$.

Proof. (a) To see that $\bar{A}$ is a sub-additive aggregation function, let $\mathbf{x}, \mathbf{z} \in \mathbb{R}_{+}^{n}$. Then

$$
\begin{aligned}
\bar{A}(\mathbf{x}+\mathbf{z}) & =\sup _{\mathbf{y} \in \mathbb{R}_{+}^{n}}(A(\mathbf{x}+\mathbf{z}+\mathbf{y})-A(\mathbf{y}))=\sup _{\mathbf{y} \in \mathbb{R}_{+}^{n}}(A(\mathbf{x}+\mathbf{z}+\mathbf{y})-A(\mathbf{z}+\mathbf{y})+A(\mathbf{z}+\mathbf{y})-A(\mathbf{y})) \\
& \leq \sup _{\mathbf{y} \in \mathbb{R}_{+}^{n}}(A(\mathbf{x}+\mathbf{z}+\mathbf{y})-A(\mathbf{z}+\mathbf{y}))+\sup _{\mathbf{y} \in \mathbb{R}_{+}^{n}}(A(\mathbf{z}+\mathbf{y})-A(\mathbf{y})) \\
& =\sup _{\mathbf{y} \geq \mathbf{z}}(A(\mathbf{x}+\mathbf{y})-A(\mathbf{y}))+\bar{A}(\mathbf{z}) \leq \sup _{\mathbf{y} \in \mathbb{R}_{+}^{n}}(A(\mathbf{x}+\mathbf{y})-A(\mathbf{y}))+\bar{A}(\mathbf{z})=\bar{A}(\mathbf{x})+\bar{A}(\mathbf{z})
\end{aligned}
$$

and thus $\bar{A}$ is a sub-additive aggregation function.

To prove (b) it is sufficient to notice that

$$
\bar{A}(\mathbf{x})=\sup _{\mathbf{y} \in \mathbb{R}_{+}^{n}}(A(\mathbf{x}+\mathbf{y})-A(\mathbf{y})) \geq\left.(A(\mathbf{x}+\mathbf{y})-A(\mathbf{y}))\right|_{\mathbf{y}=\mathbf{0}}=A(\mathbf{x})-A(\mathbf{0})=A(\mathbf{x})
$$

for all $\mathbf{x} \in \mathbb{R}_{+}^{n}$ which implies that $\bar{A} \geq A$.

(c) If $A$ is sub-additive then $A(\mathbf{x}+\mathbf{y}) \leq A(\mathbf{x})+A(\mathbf{y})$ which implies that

$$
\bar{A}(\mathbf{x})=\sup _{\mathbf{y} \in \mathbb{R}_{+}^{n}}(A(\mathbf{x}+\mathbf{y})-A(\mathbf{y})) \leq \sup _{\mathbf{y} \in \mathbb{R}_{+}^{n}}(A(\mathbf{x})+A(\mathbf{y})-A(\mathbf{y}))=\sup _{\mathbf{y} \in \mathbb{R}_{+}^{n}} A(\mathbf{x})=A(\mathbf{x}),
$$

i.e., $\bar{A} \leq A$. Combining this with part (b) of this proposition we obtain that $\bar{A}=A$ as needed.

To see $(\mathrm{d})$ notice that

$$
\begin{aligned}
\bar{A}(\alpha \mathbf{1}) & =\sup _{\mathbf{y} \in \mathbb{R}_{+}^{n}}(A(\alpha \mathbf{1}+\mathbf{y})-A(\mathbf{y}))=\sup _{\mathbf{y} \in \mathbb{R}_{+}^{n}}(A(\mathbf{y}+\alpha \mathbf{1})-A(\mathbf{y}))=\sup _{\mathbf{y} \in \mathbb{R}_{+}^{n}}(A(\mathbf{y})+\alpha A(\mathbf{1})-A(\mathbf{y})) \\
& =\sup _{\mathbf{y} \in \mathbb{R}_{+}^{n}} \alpha A(\mathbf{1})=\alpha A(\mathbf{1})=A(\alpha \mathbf{1}),
\end{aligned}
$$

i.e., the diagonal of $\bar{A}$ coincides with the diagonal of $A$ if $A$ is shift-invariant.

To prove (e), notice that

$$
\begin{aligned}
\bar{A}(\mathbf{x}+\alpha \mathbf{1}) & =\sup _{\mathbf{y} \in \mathbb{R}_{+}^{n}}(A(\mathbf{x}+\alpha \mathbf{1}+\mathbf{y})-A(\mathbf{y}))=\sup _{\mathbf{y} \in \mathbb{R}_{+}^{n}}(A(\mathbf{x}+\mathbf{y})+\alpha A(\mathbf{1})-A(\mathbf{y})) \\
& =\sup _{\mathbf{y} \in \mathbb{R}_{+}^{n}}(A(\mathbf{x}+\mathbf{y})-A(\mathbf{y}))+\alpha A(\mathbf{1})=\bar{A}(\mathbf{x})+A(\alpha \mathbf{1}) .
\end{aligned}
$$

Now using (d) we have that $A(\mathbf{1})=\bar{A}(\mathbf{1})$ and thus

$$
\bar{A}(\mathbf{x}+\alpha \mathbf{1})=\bar{A}(\mathbf{x})+\alpha \bar{A}(\mathbf{1}),
$$

i.e., $\bar{A}$ is indeed a shift-invariant aggregation function if $A$ is. Now, to prove (f) notice that

$$
\begin{aligned}
\bar{A}(\alpha \mathbf{x}) & =\sup _{\mathbf{y} \in \mathbb{R}_{+}^{n}}(A(\alpha \mathbf{x}+\mathbf{y})-A(\mathbf{y}))=\sup _{\mathbf{y} \in \mathbb{R}_{+}^{n}}(A(\alpha \mathbf{x}+\alpha \mathbf{y})-A(\alpha \mathbf{y})) \\
& =\alpha \sup _{\mathbf{y} \in \mathbb{R}_{+}^{n}}(A(\mathbf{x}+\mathbf{y})-A(\mathbf{y}))=\alpha \bar{A}(\mathbf{x})
\end{aligned}
$$

for all $\mathbf{x} \in \mathbb{R}_{+}^{n}$ and all $\alpha \geq 0$ if $A$ is positively homogeneous. 
Example 2. If we take, for the sake of exemplification, a one-dimensional aggregation function $A:[0, \infty[\rightarrow[0, \infty[$ given by

$$
A(x)= \begin{cases}x^{p}, & \text { if } x \in[0,1] \\ 1, & \text { otherwise, }\end{cases}
$$

for any $p>0$ then we obtain by item c) of Proposition 2 that $\bar{A}=A$ if $p \in] 0,1]$ since $A$ is sub-additive and

$$
\bar{A}(x)= \begin{cases}1-(1-x)^{p}, & \text { if } x \in[0,1] \\ 1, & \text { otherwise }\end{cases}
$$

if $p>1$.

Example 3. If we consider a one-dimensional aggregation function $A:[0, \infty[\rightarrow[0, \infty[$ defined by

$$
A(x)=\operatorname{sgn}(\alpha)(\exp (\alpha x)-1)
$$

where $\alpha \in \mathbb{R} \backslash\{0\}$ then we obtain by $c$ ) of Proposition 2 that $\bar{A}=A$ if $\alpha<0$ since $A$ is sub-additive. On the other hand, if $\alpha>0$, the revenue transformation of $A$ does not exist.

Example 4. Let us consider $\Omega=\{1,2\}$ and Choquet integral $\mathrm{Ch}^{\mu}: \mathbb{R}_{+}^{2} \rightarrow[0, \infty$. Then one can easily compute its revenue transformation as

$$
\overline{\mathrm{Ch}}^{\mu}\left(x_{1}, x_{2}\right)= \begin{cases}x_{1} \max \left\{\mu_{1}, \mu_{12}-\mu_{2}\right\}+x_{2} \min \left\{\mu_{2}, \mu_{12}-\mu_{1}\right\}, & \text { if } x_{1} \geq x_{2}, \\ x_{1} \min \left\{\mu_{1}, \mu_{12}-\mu_{2}\right\}+x_{2} \max \left\{\mu_{2}, \mu_{12}-\mu_{1}\right\}, & \text { if } x_{1}<x_{2}\end{cases}
$$

for all $\mathbf{x} \in \mathbb{R}_{+}^{2}$, which coincides with Choquet integral $\mathrm{Ch}^{\hat{\mu}}$ where $\hat{\mu}$ is given by

$$
\hat{\mu}_{12}=\mu_{12}, \quad \hat{\mu}_{i}=\mu_{i}+\max \left\{0, \mu_{12}-\mu_{1}-\mu_{2}\right\}
$$

for $i \in\{1,2\}$.

In general, for $n>2$, it is an open problem whether for any monotone measure $\mu$, $\overline{\mathrm{Ch}}^{\mu}=\mathrm{Ch}^{v}$ for some monotone measure $v$. Clearly, if this is the case, $v(A)=\overline{\mathrm{Ch}}\left(\mathbf{1}_{A}\right)$ for any $A \subseteq \Omega$. Note that for any $\mu, v(A)=\overline{\mathrm{Ch}}^{\mu}\left(\mathbf{1}_{A}\right)$ defines a sub-modular monotone measure.

Example 5. Consider a Choquet integral $\mathrm{Ch}^{\mu}$ on $\Omega=\{1,2,3\}$ with respect to a monotone measure $\mu$ given by

$$
\mu(A)= \begin{cases}0, & \text { if } A=\varnothing, \\ 2 / 3, & \text { if }|A| \in\{1,2\} \\ 1, & \text { if } A=\Omega .\end{cases}
$$

This monotone measure is not sub-modular and one can find that $\mathrm{Ch}^{\mu}(\mathbf{x})=\left(x_{(1)}+2 x_{(3)}\right) / 3$, i.e., $\mathrm{Ch}^{\mu}$ is an OWA (Ordered Weighted Averaging) operator [18]. Its revenue transformation is again Choquet integral $\overline{\mathrm{Ch}}^{\mu}=\mathrm{Ch}^{\hat{\mu}}$ with respect to a monotone measure $\hat{\mu}$ given by

$$
\hat{\mu}(A)= \begin{cases}0, & \text { if } A=\varnothing, \\ 2 / 3, & \text { if }|A|=1, \\ 1, & \text { if }|A| \geq 2,\end{cases}
$$

and $\mathrm{Ch}^{\hat{\mu}}=\left(x_{(2)}+2 x_{(3)}\right) / 3$; and $\hat{\mu}$ is a sub-modular monotone measure.

Example 6. Let $\Omega=\{1,2,3,4\}$ and let $\mu$ be a monotone measure on $\Omega$. Let us continue with Example 2 that we presented in [19]. Note that based on the monotone measure $\mu$ introduced there, we can construct a new monotone measure $v: 2^{\Omega} \rightarrow\left[0, \infty\left[\right.\right.$ by $v(A)=\overline{\mathrm{Ch}}^{\mu}\left(\mathbf{1}_{A}\right)$ for all $A \in 2^{\Omega}$. 
The values of the chosen monotone measure $\mu$ and the related monotone measure $v$ can be found in Table 1. Note that $v$ is a sub-modular monotone measure and also, if $\overline{\mathrm{Ch}}^{\mu}$ is Choquet integral then it would coincide with $\mathrm{Ch}^{v}$.

Table 1. Values of capacities $\mu$ and $v$ from Example 6.

\begin{tabular}{|c|c|c|c|c|c|c|c|c|c|c|c|}
\hline$A$ & $\varnothing$ & $\{1\}$ & $\{2\}$ & $\{3\}$ & $\{4\}$ & $\{1,2\}$ & $\{1,3\}$ & $\{1,4\}$ & $\{2,3\}$ & $\{2,4\}$ & $\{3,4\}$ \\
\hline$\mu(A)$ & 0 & $1 / 4$ & 0 & $1 / 4$ & 0 & $1 / 2$ & $1 / 2$ & $1 / 2$ & $1 / 2$ & $1 / 2$ & $1 / 2$ \\
\hline$v(A)$ & 0 & $1 / 2$ & $1 / 2$ & $1 / 2$ & $1 / 2$ & 1 & 1 & 1 & 1 & $1 / 2$ & 1 \\
\hline$A$ & $\{1,2,3\}$ & $\{1,2,4\}$ & $\{1,3,4\}$ & $\{2,3,4\}$ & $\{1,2,3,4\}$ & & & & & & \\
\hline$\mu(A)$ & $3 / 4$ & $1 / 2$ & $3 / 4$ & $1 / 2$ & 1 & & & & & & \\
\hline$v(A)$ & 1 & 1 & 1 & 1 & 1 & & & & & & \\
\hline
\end{tabular}

\section{Constructions of Coherent Upper Previsions}

In this section, we will construct coherent upper previsions using aggregation functions with special properties. Analogous construction process as in [4,19] will be adopted.

Proposition 3. Let $A: \mathbb{R}_{+}^{n} \rightarrow[0, \infty[$ be a sub-additive, positively homogeneous and shift-invariant aggregation function such that $A(\mathbf{1}) \neq 0$. Then the mapping cup: $\mathbb{R}^{n} \rightarrow \mathbb{R}$ given by

$$
\operatorname{cup}(\mathbf{x})=\frac{A(\mathbf{x}-(\inf \mathbf{x}) \mathbf{1})}{A(\mathbf{1})}+\inf \mathbf{x}
$$

for all $\mathbf{x} \in \mathbb{R}^{n}$ defines a coherent upper prevision.

Proof. First of all, note that for all $\mathbf{x} \in \mathbb{R}^{n}, \mathbf{x}-(\inf \mathbf{x}) \mathbf{1} \geq \mathbf{0}$, and thus the definition of cup is valid. Since $\mathbb{R}^{n}$ is a linear space to prove that cup defines a coherent upper prevision we will prove that cup obeys conditions (CUP1)-(CUP3). To see that (CUP1) holds, notice that

$$
\begin{aligned}
\operatorname{cup}(\mathbf{x}) & =\frac{A(\mathbf{x}-(\inf \mathbf{x}) \mathbf{1})}{A(\mathbf{1})}+\inf \mathbf{x} \leq \frac{A(\sup (\mathbf{x}-(\inf \mathbf{x}) \mathbf{1}) \mathbf{1})}{A(\mathbf{1})}+\inf \mathbf{x} \\
& =(\sup \mathbf{x}-\inf \mathbf{x}) \frac{A(\mathbf{1})}{A(\mathbf{1})}+\inf \mathbf{x}=\sup \mathbf{x}-\inf \mathbf{x}+\inf \mathbf{x}=\sup \mathbf{x}
\end{aligned}
$$

for all $\mathbf{x} \in \mathbb{R}^{n}$. To see (CUP2) it is enough to consider

$$
\begin{aligned}
\operatorname{cup}(\alpha \mathbf{x}) & =\frac{A(\alpha \mathbf{x}-(\inf \alpha \mathbf{x}) \mathbf{1})}{A(\mathbf{1})}+\inf \alpha \mathbf{x}=\frac{A(\alpha \mathbf{x}-\alpha(\inf \mathbf{x}) \mathbf{1})}{A(\mathbf{1})}+\alpha \inf \mathbf{x} \\
& =\alpha \frac{A(\mathbf{x}-(\inf \mathbf{x}) \mathbf{1})}{A(\mathbf{1})}+\alpha \inf \mathbf{x}=\alpha\left(\frac{A(\mathbf{x}-(\inf \mathbf{x}) \mathbf{1})}{A(\mathbf{1})}+\inf \mathbf{x}\right)=\alpha \operatorname{cup}(\mathbf{x})
\end{aligned}
$$

for all $\alpha \geq 0$ and all $\mathbf{x} \in \mathbb{R}^{n}$. Lastly, it remains to show that the condition (CUP3) holds, i.e., to show that cup is sub-additive. Because infimum is a super-additive aggregation function, we know that $\inf (\mathbf{x}+\mathbf{y}) \geq \inf \mathbf{x}+\inf \mathbf{y}$ and thus $r=\inf (\mathbf{x}+\mathbf{y})-\inf \mathbf{x}-\inf \mathbf{y} \geq 0$. Note that

$$
\operatorname{cup}(\mathbf{x}+\mathbf{y})=\frac{A(\mathbf{x}+\mathbf{y}-(\inf (\mathbf{x}+\mathbf{y})) \mathbf{1})}{A(\mathbf{1})}+\inf (\mathbf{x}+\mathbf{y})
$$

Now, from $-\inf (\mathbf{x}+\mathbf{y})=-\inf \mathbf{x}-\inf \mathbf{y}-r$, we obtain that

$$
\operatorname{cup}(\mathbf{x}+\mathbf{y})=\frac{A(\mathbf{x}+\mathbf{y}-(r+\inf \mathbf{x}+\inf \mathbf{y}) \mathbf{1})+\overbrace{r A(\mathbf{1})-r A(\mathbf{1})}^{0}}{A(\mathbf{1})}+\inf (\mathbf{x}+\mathbf{y})
$$


Using the shift-invariance of $A$ and the fact that $r \geq 0$ we have that

$$
\operatorname{cup}(\mathbf{x}+\mathbf{y})=\frac{A((\mathbf{x}-(\inf \mathbf{x}) \mathbf{1})+(\mathbf{y}-(\inf \mathbf{y}) \mathbf{1}))}{A(\mathbf{1})}-r+\inf (\mathbf{x}+\mathbf{y}) .
$$

Noticing that $\inf (\mathbf{x}+\mathbf{y})-r=\inf \mathbf{x}+\inf \mathbf{y}$ and by the sub-additivity of $A$ we finally obtain that

$$
\operatorname{cup}(\mathbf{x}+\mathbf{y}) \leq \frac{A(\mathbf{x}-(\inf \mathbf{x}) \mathbf{1})}{A(\mathbf{1})}+\inf \mathbf{x}+\frac{A(\mathbf{y}-(\inf \mathbf{y}) \mathbf{1})}{A(\mathbf{1})}+\inf \mathbf{y}=\operatorname{cup}(\mathbf{x})+\operatorname{cup}(\mathbf{y})
$$

for all $\mathbf{x}, \mathbf{y} \in \mathbb{R}^{n}$, i.e., cup is sub-additive.

Remark 1. Moreover, we may assume that $A$ is also idempotent, i.e., $A(\alpha \mathbf{1})=\alpha$ for all $\alpha \geq 0$. With the fact that $A$ is shift-invariant it is enough to consider only the fact that $A(\mathbf{1})=1$. Assuming this extra condition, the coherent upper prevision can be constructed by

$$
\operatorname{cup}(\mathbf{x})=A(\mathbf{x}-(\inf \mathbf{x}) \mathbf{1})+\inf \mathbf{x},
$$

which simplifies the construction.

Remark 2. As another remark, note that a positively homogeneous and idempotent aggregation function $A: \mathbb{R}_{+}^{n} \rightarrow\left[0, \infty\left[\right.\right.$ is fully determined by its restriction to $[0,1]^{n}$ because

$$
A(\mathbf{x})=(\sup \mathbf{x}) A\left(\frac{\mathbf{x}}{\sup \mathbf{x}}\right),
$$

for any $\mathbf{x} \in \mathbb{R}_{+}^{n} \backslash\{\mathbf{0}\}$, where $\mathbf{x} /(\sup \mathbf{x}) \in[0,1]^{n}$. Also note that the given restriction $A_{[0,1]^{n}}$ is a $[0,1]$-aggregation function and thus we can characterize all positively homogeneous, shift-invariant and idempotent aggregation functions. To authors' best knowledge, this characterization has not been published anywhere, yet.

Proposition 4. An aggregation function $A: \mathbb{R}_{+}^{n} \rightarrow[0, \infty[$ is positively homogeneous, shiftinvariant and idempotent if and only if there exists a positively homogeneous and shift-invariant $[0,1]$-aggregation function $B:[0,1]^{n} \rightarrow[0,1]$ such that $\left.A\right|_{[0,1]^{n}}=B$ and

$$
A(\mathbf{x})=(\sup \mathbf{x}) A\left(\frac{\mathbf{x}}{\sup \mathbf{x}}\right)
$$

whenever $\mathbf{x} \neq \mathbf{0}$.

Remark 3. Note that there are positively homogeneous and shift-invariant aggregation functions that are not idempotent. As a trivial example of such aggregation function we give the sum function (with $n>1$ ).

Coherent lower previsions are obtained by the conjugacy property

$$
\operatorname{clp}(\mathbf{x})=-\operatorname{cup}(-\mathbf{x})=-\frac{A(-\mathbf{x}-(\inf (-\mathbf{x})) \mathbf{1})}{A(\mathbf{1})}-\inf (-\mathbf{x})=-\frac{A(-\mathbf{x}-(\sup (\mathbf{x})) \mathbf{1})}{A(\mathbf{1})}-\sup (\mathbf{x})
$$

We can observe that if the aggregation function $A$, that is considered to define a coherent upper prevision, is linear, then $\operatorname{clp}(\mathbf{x})=-\operatorname{cup}(-\mathbf{x})$ and a linear prevision is obtained.

Coherent upper probabilities can be obtained by Proposition 3 when only indicator vectors are considered. 
The coherent upper probability of an event $E \subseteq \Omega$ is defined by

$$
\bar{P}(E)=\operatorname{cup}\left(\mathbf{1}_{E}\right)=\frac{A\left(\mathbf{1}_{E}\right)}{A(\mathbf{1})} .
$$

and the coherent lower probability is obtained by the conjugacy property,

$$
\operatorname{clp}\left(\mathbf{1}_{E}\right)=1-\operatorname{cup}\left(\mathbf{1}_{E^{c}}\right)
$$

Example 7. Let $A: \mathbb{R}_{+}^{n} \rightarrow[0, \infty[$ be an aggregation function defined by $A(\mathbf{x})=\sup \mathbf{x}$. Note that $A$ satisfies all requirements of Proposition 3 and thus $A$ can be used to construct a coherent upper prevision. This construction leads to $\operatorname{cup}(\mathbf{x})=\sup \mathbf{x}$ which is the vacuous upper prevision.

Example 8. The Choquet integral $\mathrm{Ch}^{\hat{\mu}}$ defined in Example 5 by the revenue transformation of $\mathrm{Ch}^{\mu}$ (which is not a coherent upper prevision because it is not sub-additive), is a coherent upper prevision since it is sub-additive, positively homogeneous and such that

$$
\mathrm{Ch}^{\hat{\mu}}(\mathbf{x})=\frac{x_{(2)}+2 x_{(3)}}{3} \leq x_{(3)}=\sup \mathbf{x} .
$$

Coherent upper probabilities are obtained when only indicator vectors $\mathbf{1}_{E}$, for all $E \in 2^{\Omega}$, are considered. These are given by

$$
\bar{P}\left(\mathbf{1}_{E}\right)=\operatorname{cup}\left(\mathbf{1}_{E}\right)=\frac{\operatorname{Ch}^{\hat{\mu}}\left(\mathbf{1}_{E}\right)}{\operatorname{Ch}^{\hat{\mu}}(\mathbf{1})}=\operatorname{Ch}^{\hat{\mu}}\left(\mathbf{1}_{E}\right)=\hat{\mu}(E)= \begin{cases}0, & \text { if } E=\varnothing, \\ 2 / 3, & \text { if }|E|=1, \\ 1, & \text { if }|E| \geq 2,\end{cases}
$$

In the following example an aggregation function which cannot be used to define a coherent upper prevision is given.

Example 9. Let $\Omega=\{1,2, \ldots, n\}$. The aggregation function $A(\mathbf{x})=\sum_{i=1}^{n} x_{i}^{2}$ can not be used to construct a coherent upper prevision, because it is not sub-additive; moreover the revenue transformation $\bar{A}$ does not exists as shown in Example 1.

Example 10. Let $\Omega=\{1,2, \ldots, n\}$. The aggregation function $A(\mathbf{x})=\sum_{i}^{n} x_{i}$ can be used to construct a coherent upper prevision, because it is linear and homogeneous; by Proposition 3 we obtain that $\operatorname{cup}(\mathbf{x})$ is the mean and the coherent probability of an event is defined by the counting measure, which is the Hausdorff measure of order 0 . In this setting, $\bar{A}=A$.

In the following theorem a construction of aggregation functions, which are idempotent, positively homogeneous, shift invariant and sub-additive are proposed. They are applied in the construction of coherent upper conditional previsions.

Theorem 1. Denote by $K_{n}$ the class of all $n$-ary aggregation functions on $[0, \infty[$, which are idempotent, positively homogeneous, shift invariant and sub-additive, then for any $n, k \in \mathbb{N}$, let $A \in K_{n}$ and $E_{1}, E_{2}, \ldots, E_{n}$ be non-empty subsets of $\{1,2, \ldots, k\}$ with cardinalities $k_{1}, k_{2}, \ldots, k_{n}$, respectively, so that $E_{i}=\left\{e_{i, 1}, e_{i, 2}, \ldots, e_{i, k_{i}}\right\}$, where $e_{i, 1}<\ldots<e_{i, k_{i}}, s_{i}:\{1, \ldots, k\} \rightarrow\{1, \ldots, k\}$ are permutations and $B_{i} \in K_{k_{i}}$, then the aggregation function $C$ defined by

$$
C\left(x_{1}, \ldots, x_{k}\right)=A\left(B_{1}\left(x_{s_{1}\left(e_{1,1}\right)}, \ldots, x_{s_{1}\left(e_{1, k_{1}}\right)}\right), \ldots, B_{n}\left(x_{s_{n}\left(e_{n, 1}\right)}, \ldots, x_{s_{n}\left(e_{n, k_{n}}\right)}\right)\right)
$$

belongs to $K_{k}$. 
Proof. Observe first that each function $C_{i}:[0, \infty]^{k} \rightarrow[0, \infty[$ given by

$$
C_{i}\left(x_{1}, \ldots, x_{k}\right)=B_{i}\left(x_{s_{i}\left(e_{i, 1}\right)}, \ldots, x_{s_{i}\left(e_{i, k_{i}}\right)}\right),
$$

for $i=1,2, \ldots, n$, is an aggregation function from the class $K_{k}$. Then, for any $c \in[0, \infty[$,

$$
C(\mathbf{c})=A\left(C_{1}(\mathbf{c}), \ldots, C_{n}(\mathbf{c})\right)=A(c, \ldots, c)=c,
$$

i.e., $C$ is idempotent. Similarly, one can show the positive homogeneity, shift invariantness and sub-additivity of $C$, which ensures $C \in K_{k}$.

The previous construction method can be used to define coherent upper previsions if $C(\mathbf{1}) \neq 0$.

Corollary 1. Let $A \in K_{n}$ be a weighted arithmetic mean, i.e., a linear prevision given by

$$
A\left(x_{1}, \ldots, x_{n}\right)=\sum c_{i} x_{i}
$$

and let $B_{1}, \ldots, B_{n}$ be given as in Theorem 1 . Then the convex combination

$$
C\left(x_{1}, \ldots, x_{k}\right)=\sum_{i=1}^{n} c_{i} B_{i}\left(x_{e_{i, 1}}, \ldots, x_{e_{i, k_{i}}}\right)
$$

belongs to $K_{k}$.

Also multi-step Choquet integrals [20-22], which are multi-step aggregation functions and which are not Choquet integrals, in general, can be used to construct coherent upper previsions.

Example 11. Let $n=2, k=3, A=\max , B_{1}\left(x_{1}, x_{2}\right)=\left(\min \left\{x_{1}, x_{2}\right\}+2 \max \left\{x_{1}, x_{2}\right\}\right) / 3$, and $B_{2}\left(x_{3}\right)=x_{3}$ then

$$
C\left(x_{1}, x_{2}, x_{3}\right)=\max \left\{\frac{\min \left\{x_{1}, x_{2}\right\}+2 \max \left\{x_{1}, x_{2}\right\}}{3}, x_{3}\right\},
$$

and $C$ belongs to $K_{3}$ and $C(\mathbf{1})=1 \neq 0$ hence it can be used, by Proposition 1 and Remark 1 , to build the following coherent upper prevision

$$
\operatorname{cup}\left(x_{1}, x_{2}, x_{3}\right)=\frac{C\left(x_{1}, x_{2}, x_{3}\right)}{C(\mathbf{1})}=\max \left\{\frac{\min \left\{x_{1}, x_{2}\right\}+2 \max \left\{x_{1}, x_{2}\right\}}{3}, x_{3}\right\},
$$

Note that $A, B_{1}$ and $B_{2}$ are OWA operators and thus Choquet integrals, but $C$ cannot be expressed as a Choquet integral.

\section{Conclusions}

In this paper a construction method, based on sub-additive positively homogeneous and shift-invariant aggregation functions is proposed to define coherent upper previsions. Moreover a sub-additive transformation of an aggregation function, named revenue transformation is introduced to obtain a sub-additive aggregation function. Under some mild additional constraints, this transformed aggregation function is then used to define a coherent upper prevision. As a distinguished example one can recall the Choquet integral. If the considered monotone measure $\mu$ is sub-modular then the related asymmetric Choquet integral $\mathrm{Ch}^{\mu}$ is a coherent upper prevision. If $\mu$ is not sub-modular, clearly, $\mathrm{Ch}^{\mu}$ is not a coherent upper prevision, but based on our proposal one can define a coherent upper prevision $\overline{\mathrm{Ch}}^{\mu}$. It is an interesting open problem whether the operator $\overline{\mathrm{Ch}}^{\mu}$ is comonotone additive and thus it can be represented as a Choquet integral $\mathrm{Ch}^{v}$, where $v$ is a sub-modular monotone measure given by $v(E)=\overline{\mathrm{Ch}}^{\mu}\left(\mathbf{1}_{E}\right)$. 
We have considered finite $\sigma$-algebras $\mathcal{A}$ only, being inspired by the motivation and basic examples of coherent upper (lower) previsions known from the literature. In our future work, we aim to focus on constructions of coherent upper (lower) previsions on general measurable spaces $(\Omega, \mathcal{A})$.

Author Contributions: The contribution of the authors is equal. All authors have read and agreed to the published version of the manuscript.

Funding: This research received funding from Slovak Research and Development Agency (APVV) and from Ministry of Education, Science, Research and Sport of the Slovak Republic (VEGA).

Acknowledgments: R.M. and A.Š. were supported by the Slovak Research and Development Agency under the contracts no. APVV-17-0066 and no. APVV-18-0052. Also the support of the grant VEGA $1 / 0006 / 19$ is kindly announced.

Conflicts of Interest: The authors declare no conflict of interest.

\section{References}

1. de Finetti, B. Theory of Probability; Wiley: London, UK, 1974.

2. Doria, S. Preference orderings represented by coherent upper and lower previsions. Theory Decis. 2019, 87, 233-259. [CrossRef]

3. Walley, P. Statistical Reasoning with Imprecise Probabilities; Chapman and Hall: London, UK, 1991.

4. Doria, S.; Mesiar, R.; Šeliga, A. Construction method of coherent lower and upper previsions based on collection integrals. Boll. Dell'Unione Mat. Ital. 2020, 3, 469-476, doi:10.1007/s40574-020-00220-1. [CrossRef]

5. Beliakov, G.; Pradera, A.; Calvo, T. Aggregation Functions: a Guide for Practitioners; Springer: Heidelberg, Germany, 2007; ISBN 978-3-540-73720-9.

6. Grabisch, M.; Marichal, J.L.; Mesiar, R.; Pap, E. Aggregation Functions; Cambridge University Press: Cambridge, UK, 2009. ISBN 978-0-521-51926-7.

7. Greco, S.; Mesiar, R.; Rindone, F.; Šipeky, L. Superadditive and subadditive transformations of integrals and aggregation functions. Fuzzy Sets Syst. 2016, 291, 40-53, doi:10.1016/j.fss.2015.08.006. [CrossRef]

8. Hriňáková, K.; Šeliga, A. Remarks on super-additive and sub-additive transformations of aggregation functions. Tatra Mt. Math. Publ. 2018, 72, 55-66, doi:10.2478/tmmp-2018-00. [CrossRef]

9. Kouchakinejad, F.; Šipošová, A.; Širáň, J. Aggregation functions with given super-additive and sub-additive transformations. Int. J. Gen. Syst. 2017, 46, 225-234, doi:10.1080/03081079.2017.1300586. [CrossRef]

10. Šipošová, A. A note on the superadditive and the subadditive transformations of aggregation functions. Fuzzy Sets Syst. 2016, 299, 98-104, doi:10.1016/j.fss.2015.10.008. [CrossRef]

11. Aczél, J.; Gronau, D.; Schwaiger, J. Increasing solutions of the homogeneity equation and of similar equations. J. Math. Anal. Appl. 1994, 182, 436-464, doi:10.1006/jmaa.1994.1097. [CrossRef]

12. Candeal, J.C.; Induráin, E. Comparison meaningful operators and ordinal invariant preferences. J. Math. Anal. Appl. 2015, 432, 806-819, doi:10.1016/j.jmaa.2015.07.017. [CrossRef]

13. Denneberg, D. Non-Additive Measure and Integral; Kluwer Academic: Dordrecht, The Netherlands, 1994; ISBN 978-90-481-4404-4.

14. Wang, Z.; Yan, J. A selective overview of applications of Choquet integrals. In Asymptotic Theory in Probability and Statistics with Applications, Advanced Lectures in Mathematics; International Press of Boston: Somerville, MA, USA, 2008; Volume 2, pp. 484-514.

15. Yan, J. A short presentation of Choquet integral. In Recent Development in Stochastic Dynamics and Stochastic Analysis. Interdisciplinary Mathematical Sciences; World Scientific Publishing: Hackensack, NJ, USA, 2010; Volume 8, pp. 269-291, doi:10.1142/9789814277266_0017. [CrossRef]

16. Choquet, G. Theory of capacities. Ann. L'Institut Fourier 1954, 5, 131-295, doi:10.5802/aif.53. [CrossRef]

17. Doria, S. Characterization of a coherent upper conditional prevision as the Choquet integral with respect to its associated Hausdorff outer measure. Ann. Oper. Res. 2012, 195, 33-48, doi:10.1007/s10479-011-0899-y. [CrossRef]

18. Yager, R.R. On ordered weighted averaging aggregation operators in multicriteria decision making. IEEE Trans. Syst. Man. Cybern. 1988, 18, 183-190, doi:10.1109/21.87068. [CrossRef]

19. Doria, S.; Mesiar, R.; Šeliga, A. Integral representation of coherent lower previsions by super-additive integrals. Axioms 2020, 9 , 43, doi:10.3390/axioms9020043. [CrossRef]

20. Mesiar, R.; Vivona, D. Two step integral with respect to fuzzy measure. Tatra Mt. Math. Publ. 1999, 16, 359-368.

21. Murofushi, T.; Narukawa, Y. A characterization of multi-level descrete Choquet integral on a finite set. In Proceedings of the 7th Workshop on Evaluation of Heard and Mind, Kitakyushu, Japan, 8-9 November 2002. (In Japanese)

22. Narukawa, Y.; Torra, V. Twofold integral and Multi-step Choquet intgral. Kybernetika 2004, 40, 39-50. 